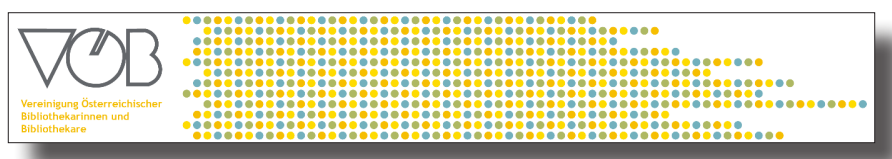

\title{
STATUTEN DER VEREINIGUNG ÖSTERREICHISCHER BIBLIOTHEKARINNEN UND BIBLIOTHEKARE
}

\section{§ 1. Name, Sitz und Tätigkeitsbereich}

1) Der Verein führt den Namen „Vereinigung Österreichischer Bibliothekarinnen und Bibliothekare".

2) Der Verein hat seinen Sitz in Graz.

3) Der Verein erstreckt seine Tätigkeit über das gesamte Bundesgebiet Österreichs.

\section{§ 2. Zweck und Ziele}

Die Vereinsziele sind:

a) die Förderung des österreichischen Bibliotheks-, Informations- und Dokumentationswesens

b) die intensive Information der Öffentlichkeit über die Bedeutung und die Aufgaben des Bibliotheks-, Informations- und Dokumentationswesens

c) die Zusammenarbeit mit in- und ausländischen Berufsvereinigungen und internationalen Fachorganisationen

d) die Ausarbeitung (Erstellung) fachlicher Empfehlungen und Anleitungen

e) die Vertretung der Interessen der Mitglieder des Vereins im In- und Ausland

f) die Förderung der beruflichen Aus- und Fortbildung der Mitglieder

g) die Förderung von besonders engagierten Berufskolleginnen und -kollegen durch Stipendien, die Vergabe von Auftragsarbeiten, die Unterstützung von Projekten etc. Um das Bibliotheks-, Informations- und Dokumentationswesen verdiente Persönlichkeiten können durch die Verleihung von Ehrenzeichen geehrt werden. 


\section{$\S 3$. Tätigkeiten und Art der Aufbringung finanzieller Mittel, die zur Ver- wirklichung des Vereinszweckes vorgesehen sind}

1) Der Vereinszweck soll durch die Organisation von für das Bibliotheks-, Informations- und Dokumentationswesen relevanten Veranstaltungen, die Herausgabe einschlägiger Publikationen und sonstige den Vereinszweck fördernde Maßnahmen erreicht werden.

2) Die zur Verwirklichung des Vereinszweckes erforderlichen finanziellen Mittel werden aufgebracht durch: Mitgliedsbeiträge, Tagungs- und Seminarbeiträge, Verkauf von vereinseigenen Publikationen, Spenden, Subventionen, sonstige zweckdienliche Zuwendungen.

\section{§ 4. Arten der Mitgliedschaft}

1) Die Mitglieder des Vereins gliedern sich in:

1. Ordentliche Mitglieder

2. Außerordentliche Mitglieder

3. Unterstützende Mitglieder

4. Ehrenmitglieder

2) Ordentliche Mitglieder können alle aktiven oder im Ruhestand befindlichen Mitarbeiter/innen an Einrichtungen des Bibliotheks-, Informations- und Dokumentationswesens sein.

3) Außerordentliche Mitglieder können natürliche Personen sein, die nicht die Voraussetzungen für die ordentliche Mitgliedschaft nachweisen können, aber bereit sind, die Vereinsziele aktiv zu fördern.

4) Unterstützende Mitglieder können natürliche und juristische Personen sein, die die Vereinsziele durch die Leistung eines mehrfachen Mitgliedsbeitrages unterstützen.

5) Zu Ehrenmitgliedern werden Personen in Würdigung ihrer besonderen Verdienste um den Verein oder das Bibliotheks-, Informationsund Dokumentationswesen ernannt.

\section{$\S 5$. Erwerb der Mitgliedschaft}

1) Der Antrag um Aufnahme in den Verein ist beim Sekretariat einzubringen.

2) Über die Aufnahme entscheidet der Vorstand mit einfacher Mehrheit im Wege eines Umlaufbeschlusses, wobei die Zustimmung als erteilt gilt, wenn nicht binnen einer Woche begründete Einwände von 
einem Vorstandsmitglied vorgebracht werden. Diesfalls obliegt die Entscheidung über die Aufnahme der nächsten Vorstandssitzung.

3) Die Ernennung zum Ehrenmitglied erfolgt auf Vorschlag des Vorstandes durch die Generalversammlung.

\section{$\S$ 6. Beendigung der Mitgliedschaft}

1) Die Mitgliedschaft endet durch Tod, durch freiwilligen Austritt, durch Ausschluss und bei juristischen Personen auch durch Verlust der Rechtspersönlichkeit.

2) Der freiwillige Austritt kann jederzeit erfolgen; er ist dem Vorstand schriftlich mitzuteilen und wird mit dem Tag der Postaufgabe der Mitteilung wirksam. Er entbindet nicht von der Entrichtung des Jahresbeitrages für das laufende Kalenderjahr.

3) Wer trotz zweifacher schriftlicher Mahnung den Beitrag für das vergangene Kalenderjahr nicht entrichtet hat, kann vom Vorstand ausgeschlossen werden.

4) Der Ausschluss eines Mitgliedes wegen grober Verletzung der Mitgliedspflichten oder wegen den Interessen des Vereins widersprechenden Verhaltens ist vom Vorstand mit Zweidrittelmehrheit zu beschließen. Gegen einen solchen Beschluss ist eine Berufung an die Generalversammlung zulässig. Die Berufung ist innerhalb eines Monats schriftlich beim Vorstand einzubringen. Der Berufung kommt keine aufschiebende Wirkung zu. Über die Berufung entscheidet die Generalversammlung mit einfacher Mehrheit.

5) Die Aberkennung der Ehrenmitgliedschaft kann wegen der gleichen Gründe wie in $\S 6$ (4) von der Generalversammlung mit Zweidrittelmehrheit beschlossen werden.

\section{$\S 7$. Rechte und Pflichten der Mitglieder}

1) Die Mitglieder sind berechtigt, an allen Veranstaltungen des Vereins teilzunehmen und die Publikationen des Vereins, soweit deren Bezug nicht durch den Mitgliedsbeitrag gedeckt ist, zu einem ermäßigten Preis zu beziehen. Das Stimmrecht in der Generalversammlung steht allen Mitgliedern, die den Mitgliedsbeitrag für das laufende Kalenderjahr entrichtet haben, zu. Das passive Wahlrecht steht nur den ordentlichen Mitgliedern und den Ehrenmitgliedern zu.

2) Die Mitglieder sind verpflichtet, die Ziele und Interessen des Vereins nach Kräften zu fördern und zu unterstützen und alles zu un- 
terlassen, wodurch das Ansehen und der Zweck des Vereins leiden könnte. Sie haben weiters die Vereinsstatuten und die Beschlüsse der Vereinsorgane zu beachten und sind zur Bezahlung der Mitgliedsbeiträge in der von der Generalversammlung festgesetzten Höhe verpflichtet.

3) Ordentliche Mitglieder sind nach (mindestens) vierzigjähriger Mitgliedschaft von der Zahlung des Mitgliedsbeitrags befreit.

4) Ordentliche Mitglieder, die sich in einem Kalenderjahr mindestens sechs Monate in Mutterschutz, Karenz oder Väterkarenz befinden sind (auf Antrag) für dieses Kalenderjahr von der Zahlung des Mitgliedsbeitrags befreit. Während dieses Zeitraums ruhen Ansprüche auf Ermäßigungen, die sich aus der Mitgliedschaft ergeben.

5) Ehrenmitglieder sind von der Pflicht zur Entrichtung des Mitgliedsbeitrages befreit.

\section{§ 8. Vereinsorgane}

Organe des Vereins sind:
1. die Generalversammlung
2. das Präsidium
3. der Vorstand
4. die Rechnungsprüfung
5. das Schiedsgericht
6. die Kommissionen
7. die Arbeitsgruppen

\section{§ 9. Die Generalversammlung}

1) Eine ordentliche Generalversammlung findet alle zwei Jahre, nach Möglichkeit in Zusammenhang mit dem „Österreichischen Bibliothekartag", statt.

2) Eine außerordentliche Generalversammlung ist einzuberufen:
a) auf Beschluss des Vorstandes
b) auf Beschluss der ordentlichen Generalversammlung
c) auf schriftlichen Antrag von mindestens einem Zehntel aller Mit- glieder
d) auf Verlangen eines Rechnungsprüfers

3) Die außerordentliche Generalversammlung muss auf einen Termin binnen vier Wochen nach Beschlussfassung bzw. Einlangen des Verlangens angesetzt werden. 
4) Der Termin der Generalversammlung muss vierzehn Tage im Voraus unter Angabe der Tagesordnung schriftlich, per Fax oder per E-Mail allen Mitgliedern mitgeteilt werden.

5) Die Generalversammlung ist bei statutengemäßer Einberufung ohne Rücksicht auf die Anzahl der anwesenden stimmberechtigten Mitglieder beschlussfähig. Die Übertragung des Stimmrechts im Wege einer schriftlichen Bevollmächtigung eines stimmberechtigten Mitgliedes ist zulässig.

6) Anträge zur Tagesordnung sind schriftlich, per Fax oder E-Mail spätestens acht Tage vor dem Termin der Generalversammlung beim Sekretariat einzubringen.

7) Unmittelbar vor der Generalversammlung ist eine Vorstandssitzung abzuhalten.

8) Die Generalversammlung wird von der Präsidentin/dem Präsidenten, bei Verhinderung von der ersten Stellvertreterin/dem ersten Stellvertreter, bei deren/dessen Verhinderung von der zweiten Stellvertreterin/dem zweiten Stellvertreter einberufen und geleitet.

9) Bei jeder Generalversammlung ist ein Protokoll zu führen, das von der Schriftführerin/dem Schriftführer und der Präsidentin/dem Präsidenten zu unterzeichnen ist.

\section{$\S 10$. Aufgaben der Generalversammlung}

1) Der Generalversammlung sind folgende Aufgaben vorbehalten:
a) Entgegennahme und Genehmigung des Berichtes des Präsidiums und des Rechenschaftsberichtes der Rechnungsprüfer und des Rechnungsabschlusses

b) Entlastung der Mitglieder des Präsidiums

c) Wahl der Präsidentin/des Präsidenten und der beiden Stellvertreterinnen/Stellvertreter

d) Wahl der Mitglieder des Vorstandes

e) Wahl der Rechnungsprüfer/innen und deren Stellvertreter/innen

f) Enthebung der Mitglieder des Präsidiums, der Mitglieder des Vorstandes und der Rechnungsprüfer/innen

g) Verleihung und Aberkennung der Ehrenmitgliedschaft und der Ehrenzeichen

h) Entscheidung über Berufungen gegen den Beschluss betreffend Nichtaufnahme als Mitglied und Aberkennung der Mitgliedschaft

i) Beschlussfassung über die Statuten (2/3 Mehrheit) und die Geschäftsordnung (2/3 Mehrheit) des Vereins, wobei dies jedenfalls in der Tagesordnung enthalten sein muss 
j) Beschlussfassung über die Höhe des Mitgliedsbeitrages

k) Beschlussfassung über die von den Mitgliedern über das Sekretariat oder vom Vorstand eingebrachten Anträge. In der Generalversammlung vorgebrachte mündliche Anträge können behandelt werden, wenn ein Drittel der anwesenden stimmberechtigten Mitglieder einen solchen Antrag unterstützt.

I) Beratung und Beschlussfassung über sonstige auf der Tagesordnung stehende Themen.

m) Beschlussfassung über die freiwillige Auflösung des Vereins (2/3 Mehrheit).

n) Entgegennahme von allfälligen Berichten des Schiedsgerichtes.

2) Die Generalversammlung fasst ihre Beschlüsse - soweit nichts anderes angegeben - mit einfacher Mehrheit.

\section{$\S$ 11. Das Präsidium}

1) Das Präsidium besteht aus:

a) der Präsidentin/dem Präsidenten

b) den zwei Stellvertreterinnen/Stellvertretern

c) der Sekretärin/dem Sekretär

d) der Kassiererin/dem Kassier

e) der Schriftführerin/dem Schriftführer

f) den höchstens vier Beirätinnen/Beiräten

2) Die Präsidentin/der Präsident und die beiden Stellvertreterinnen/ Stellvertreter werden auf die Dauer von zwei Jahren von der Generalversammlung gewählt. Die übrigen Mitglieder des Präsidiums werden auf Vorschlag der Präsidentin/des Präsidenten vom Vorstand gewählt. Die Funktionsperiode des Präsidiums dauert jedenfalls bis zur Wahl des neuen Präsidiums. Auf Beschluss der Generalversammlung kann die Funktionsperiode des Präsidiums auf einen jeweils kürzeren Zeitraum festgelegt werden, wobei die Funktionsperiode des Präsidiums an jene des Vorstands ( $\$ 12$ Abs. 2) gekoppelt ist.

3) Das Präsidium ist von der Präsidentin/dem Präsidenten, bei Verhinderung von einer Stellvertreterin/einem Stellvertreter schriftlich, per Fax, per E-Mail oder mündlich einzuberufen und zu leiten.

4) Das Präsidium ist beschlussfähig, wenn mindestens die Hälfte aller Mitglieder anwesend ist.

5) Das Präsidium fasst seine Beschlüsse mit einfacher Mehrheit. Bei Stimmengleichheit gibt die Stimme der Präsidentin/des Präsidenten, bei deren/dessen Verhinderung ihrer Stellvertreterin/seines Stellver- 
treters den Ausschlag. Bei geheimer Abstimmung gilt bei Stimmengleichheit der Antrag als abgelehnt.

6) In dringenden Fällen kann, wenn die Abhaltung einer Sitzung aus organisatorischen Gründen nicht möglich ist, das Präsidium auch Umlaufbeschlüsse mit einfacher Mehrheit fassen. Ein solcher Beschluss ist gültig, wenn mindestens 2/3 der Mitglieder des Präsidiums an der Abstimmung teilgenommen haben.

7) Die Präsidentin/der Präsident (oder im Einzelfall eine Beauftragte/ ein Beauftragter) vertritt den Verein nach außen (mit Ausnahme von Abs. 12) und unterfertigt alle Schriftstücke. Ihr/lhm obliegt die Einberufung und Leitung der Sitzungen des Präsidiums, des Vorstandes, der Generalversammlungen.

8) Den Stellvertreterinnen/Stellvertretern obliegt die Vertretung der Präsidentin/des Präsidenten in allen Angelegenheiten.

9) Der Sekretärin/dem Sekretär obliegt die interne Kommunikation und die Führung der Korrespondenz und der Mitgliederliste.

10) Die Schriftführerin/der Schriftführer hat die Sitzungsprotokolle zu führen.

11) Sekretärin/Sekretär und Schriftführerin/Schriftführer vertreten sich gegenseitig.

12) Die Kassiererin/der Kassier ist für die ordnungsgemäße Geldgebarung des Vereins verantwortlich und hat der ordentlichen Generalversammlung den Kassenbericht zu erstatten.

13) Die Kassiererin/der Kassier vertritt den Verein in finanziellen Angelegenheiten nach außen. Insbesondere obliegen ihr/ihm die Führung der Konten, die Einhebung der Beiträge und die Tätigung von Überweisungen. Im Fall der Verhinderung wird die Kassiererin/der Kassier von der Präsidentin/dem Präsident und bei deren/dessen Verhinderung von den Stellvertreterinnen und Stellvertretern der Präsidentin/ des Präsidenten vertreten.

14) Die Beirätinnen/Beiräte haben die Präsidentin/den Präsidenten und deren Stellvertreterinnen/Stellvertreter in ihrer Tätigkeit zu unterstützen.

\section{$\S 12$. Der Vorstand}

1) Der Vorstand besteht aus allen Mitgliedern des Präsidiums, den Vorsitzenden der Kommissionen für die Dauer ihrer Funktion, je einer Vertreterin/einem Vertreter verwandter Berufsvereinigungen Österreichs, mit denen ein entsprechendes Abkommen besteht, und 
weiteren bis zu zwölf von der Generalversammlung zu wählenden Vereinsmitgliedern.

2) Die Mitglieder des Vorstandes werden auf die Dauer von zwei Jahren gewählt. Eine Wiederwahl ist zulässig. Auf Beschluss der Generalversammlung kann die Funktionsperiode des Vorstands auf einen jeweils kürzeren Zeitraum festgelegt werden, wobei die Funktionsperiode des Vorstands an jene des Präsidiums ( $§ 11$ Abs. 2) gekoppelt ist.

3) Der Vorstand ist bei Anwesenheit von mindestens einem Drittel der Mitglieder, von denen mindestens 3 dem Präsidium angehören müssen, beschlussfähig.

4) Der Vorstand ist von der Präsidentin/dem Präsidenten, bei Verhinderung von einer Stellvertreterin/einem Stellvertreter schriftlich, per Fax, per E-Mail oder mündlich einzuberufen und zu leiten.

5) Der Vorstand fasst seine Beschlüsse mit einfacher Mehrheit. Bei Stimmengleichheit gibt die Stimme der Präsidentin/des Präsidenten, bei deren/dessen Verhinderung ihrer Stellvertreterin/seines Stellvertreters den Ausschlag. Bei geheimer Abstimmung gilt bei Stimmengleichheit der Antrag als abgelehnt.

6) In dringenden Fällen kann, wenn die Abhaltung einer Sitzung aus organisatorischen Gründen nicht möglich ist, der Vorstand auch Umlaufbeschlüsse mit einfacher Mehrheit fassen. Ein solcher Beschluss (ausgenommen die Aufnahme neuer Mitglieder nach $\S 5$ Abs. 2) ist gültig, wenn mindestens 2/3 der Mitglieder des Vorstands an der Abstimmung teilgenommen haben.

\section{$\S 13$. Aufgaben des Vorstandes}

1) Der Vorstand führt gemeinsam mit dem Präsidium die laufenden Geschäfte des Vereins und hat jene Beschlüsse zu fassen, die nicht der Generalversammlung vorbehalten sind.

2) Insbesondere obliegt dem Vorstand:

1. die Neuaufnahme und der Ausschluss von Mitgliedern

2. die Kenntnisnahme und Genehmigung der Aktivitäten des Präsidiums

3. Die Wahl der nicht von der Generalversammlung gewählten Präsidiumsmitglieder auf Vorschlag der Präsidentin/des Präsidenten 4. die Einsetzung von Kommissionen zur Durchführung bestimmter Aufgaben

5. die Bestellung der Vorsitzenden/des Vorsitzenden einer Kommission auf Vorschlag der Kommissionsmitglieder 
6. die Entscheidung über den Fortbestand einer Kommission

7. die Einsetzung von Arbeitsgruppen zur Lösung von Einzelfragen

8. die Planung, Durchführung und Koordinierung aller Aktivitäten der Vereinigung, wobei auf die finanziellen Auswirkungen besonders Bedacht zu nehmen ist

9. die Erstattung von Empfehlungen und das Einbringen von Anträgen für Beschlüsse der Generalversammlung

10. die Genehmigung von Aufwandsentschädigungen

11. die Übertragung der Durchführung von Aufgaben an einzelne Mitglieder oder Personengruppen der Vereinigung.

\section{$\S$ 14. Die Rechnungsprüfung}

1) Die beiden Rechnungsprüferinnen/Rechnungsprüfer und ihre beiden Stellvertreter/innen werden auf die Dauer von zwei Jahren von der Generalversammlung gewählt. Eine Wiederwahl ist zulässig.

2) Die beiden Rechnungsprüferinnen/Rechnungsprüfer müssen ordentliche Vereinsmitglieder sein und dürfen nicht dem Vorstand angehören.

3) Den Rechnungsprüferinnen/Rechnungsprüfern obliegt die laufende Kontrolle der finanziellen Gebarung des Vereins und die Überprüfung des jährlichen Rechnungsabschlusses.

4) Jede Rechnungsprüferin/jeder Rechnungsprüfer hat das Recht, schriftlich eine außerordentliche Generalversammlung zu beantragen.

\section{$\S$ 15. Das Schiedsgericht}

1) In allen aus dem Vereinsverhältnis entstehenden Streitigkeiten entscheidet das Schiedsgericht.

2) Das Schiedsgericht setzt sich aus drei ordentlichen Vereinsmitgliedern zusammen. Jeder Streitteil wählt eine Schiedsrichterin/einen Schiedsrichter. Diese wählen ein drittes Vereinsglied als Vorsitzende/ Vorsitzenden. Bei Stimmengleichheit entscheidet das Los unter den Vorgeschlagenen.

3) Das Schiedsgericht muss vor seiner Entscheidung beiden Streitparteien ausreichend Gehör gewähren. Es fällt seine Entscheidung bei Anwesenheit aller Mitglieder mit einfacher Mehrheit. Bei Stimmengleichheit entscheidet die/der Vorsitzende. Die Entscheidungen des Schiedsgerichtes sind vereinsintern endgültig. 


\section{§ 16. Die Kommissionen}

1) Für die Bearbeitung von Fachfragen kann der Vorstand bis auf Widerruf Kommissionen einsetzen.

2) Kommissionsmitglieder können nur Vereinsmitglieder mit einschlägiger Erfahrung sein.

3) Die/der Vorsitzende einer Kommission wird auf Vorschlag der Kommissionsmitglieder vom Vorstand bestellt.

4) Über die Tätigkeit der Kommission ist dem Vorstand zu berichten.

5) Der Vorstand entscheidet über den Fortbestand der Kommission oder deren Auflösung.

\section{$\S 17$. Arbeitsgruppen}

1) Zur Lösung fachspezifischer Einzelaufgaben können vom Vorstand Arbeitsgruppen eingesetzt werden.

2) Jede Kommission kann zur Lösung von Einzelaufgaben Arbeitsgruppen einsetzen. Dies bedarf der Genehmigung des Vorstandes.

3) Die Arbeitsgruppen haben der Kommission Bericht zu erstatten. Die Kommission oder der Vorstand stellen die Erfüllung der Einzelaufgabe fest und entscheiden über die Auflösung der Arbeitsgruppe.

\section{$\S$ 18. Freiwillige Auflösung des Vereins}

1) Die freiwillige Auflösung des Vereins kann nur in einer zu diesem Zweck einberufenen Generalversammlung und nur mit Zweidrittelmehrheit der abgegebenen gültigen Stimmen beschlossen werden.

2) Die Generalversammlung hat auch - sofern Vereinsvermögen vorhanden ist - über die Liquidation zu beschließen. Sie muss eine Liquidatorin/ einen Liquidator berufen und beschließen, wem das nach Abdeckung der Passiva verbleibende Vermögen zu übertragen ist.

3) Das verbleibende Vermögen soll, soweit dies möglich und erlaubt ist, einer Organisation zufallen, die gleiche oder ähnliche Zwecke wie die Vereinigung Österreichischer Bibliothekarinnen und Bibliothekare verfolgt.

4) Es darf in keiner wie immer gearteten Form den Vereinsmitgliedern zugute kommen.

Wien, am 20. Februar 2018 